DEMONSTRATIO MATHEMATICA

Vol. XXVIII No $2 \quad 1995$

B. G. Pachpatte

\title{
ON HARDY TYPE INTEGRAL INEQUALITIES FOR FUNCTIONS OF TWO VARIABLES
}

\section{Introduction}

In the course of attempts to simplify the proof of Ililbert's double series theorem [3] (p. 226), Hardy [2] first proved in 1920 the most famous integral inequality which is now known in the literature as Hardy's inequality [3] (p. 240). Many papers (see [1]-[6]) deal with the alternative proofs and various generalizations of Hardy's integral inequality. In particular, in [4] are proved some interesting variants of Hardy's integral inequality given in [3] (p. 240). The purpose of this paper is to establish some new integral inequalities of Hardy's type involving functions of two independent variables which claim their origin to a certain variant of Hardy's inequality given in [4] (Theorem 2) and to our recent results given in [6]. Our proofs are simple and based on the applications of the well known Fubini's theorem.

\section{Statement of results}

Throughout the paper without further mention, we assume that all the integrals exist on the respective domains of their definitions.

Our main result is given in the following theorem.

ТнЕОRЕм 1. Let $f$ be a nonnegative integrable function on $\Delta=(0, a) \times$ $\times(0, b)$, where $a, b$ are positive constants, and $r_{1}, r_{2}$ be positive and absolutely continuous functions on $(0, \infty)$ such that

$$
\begin{aligned}
& 1+\frac{p x}{p-1} \frac{r_{1}^{\prime}(x)}{r_{1}(x)} \geq \frac{1}{\alpha}, \\
& 1+\frac{p y}{p-1} \frac{r_{2}^{\prime}(y)}{r_{2}(y)} \geq \frac{1}{\beta}
\end{aligned}
$$

AMS (1991) subject classification: 26D15, 26D20.

Key words and phrases: Hardy type integral inequalities, functions of two variables, Fubini's theorem, Hölder's inequality. 
for almost all $x, y \in(0, \infty)$ and for some constants $\alpha>0, \beta>0, p>1$. If $F$ is defined by

$$
F(x, y)=\frac{1}{r_{1}(x) r_{2}(y)} \int_{x / 2}^{x} \int_{y / 2}^{y} \frac{r_{1}(s) r_{2}(t) f(s, t)}{s t} d t d s, \quad(x, y) \in \Delta
$$

then

$$
\begin{aligned}
& \int_{0}^{a} \int_{0}^{b}\left\{\frac{F(x, y)}{x y}\right\}^{p} d y d x \leq(\alpha \beta)^{p}\left(\frac{p}{p-1}\right)^{2 p} \int_{0}^{a} \int_{0}^{b}\left\{\frac{1}{x y r_{1}(x) r_{2}(y)} \times\right. \\
& \quad \times \mid r_{2}(y)\left(r_{1}(x) f(x, y)-r_{2}(x / 2) f(x / 2, y)\right)-r_{2}(y / 2)\left(r_{1}(x) f(x, y / 2)\right. \\
& \left.\left.-r_{1}(x / 2) f(x / 2, y / 2)\right) \mid\right\}^{p} d y d x .
\end{aligned}
$$

We next establish the following slightly different versions of Theorem 1 which, we believe, are of independent interest.

THEOREM 2. Let $g$ be a nonnegative integrable function on $\Delta$ and the functions $r_{1}, r_{2}$ be as defined in Theorem 1. If $G$ is defined by

$$
G(x, y)=\frac{1}{r_{1}(x) r_{2}(y)} \int_{x / 2}^{x} \int_{0}^{y} \frac{r_{1}(s) r_{2}(t) g(s, t)}{s t} d t d s, \quad(x, y) \in \Delta,
$$

then

$$
\begin{aligned}
& \int_{0}^{a} \int_{0}^{b}\left\{\frac{G(x, y)}{x y}\right\}^{p} d y d x \\
& \leq(\alpha \beta)^{p}\left(\frac{p}{p-1}\right)^{2 p} \int_{0}^{a} \int_{0}^{b}\left\{\frac{1}{x y r_{1}(x)} \mid r_{1}(x) g(x, y)\right. \\
&\left.\quad-r_{1}(x / 2) g(x / 2, y) \mid\right\}^{p} d y d x,
\end{aligned}
$$

for $p>1$.

THEOREM 3. Let $h$ be a nonnegative integrable function on $\Delta$ and the functions $r_{1}, r_{2}$ be as defined in Theorem 1 . If $H$ is defined by

$$
H(x, y)=\frac{1}{r_{1}(x) r_{2}(y)} \int_{0}^{x} \int_{y / 2}^{y} \frac{r_{1}(s) r_{2}(t) h(s, t)}{s t} d t d s, \quad(x, y) \in \Delta,
$$

then

$$
\int_{0}^{a} \int_{0}^{b}\left\{\frac{H(x, y)}{x y}\right\}^{p} d y d x
$$

$\leq(\alpha \beta)^{p}\left(\frac{p}{p-1}\right)^{2 p} \int_{0}^{a} \int_{0}^{b}\left\{\frac{1}{x y r_{2}(y)}\left|r_{2}(y) h(x, y)-r_{2}(y / 2) h(x, y / 2)\right|\right\}^{p} d y d x$ for $p>1$. 
Re mark 1. The inequalities (4), (6), (8) extend our result in [6] (Theorem 1 with $p=m$ therein) to the case of two independent variables. In the special case of $r_{1}(x)=r_{2}(y)=1$, inequalities (4), (6) and (8) reduce to

$\int_{0}^{a} \int_{0}^{b}\left\{\frac{F(x, y)}{x y}\right\}^{p} d y d x$

$\leq\left(\frac{p}{p-1}\right)^{2 p} \int_{0}^{a} \int_{0}^{b}\left\{\frac{1}{x y}|f(x, y)-f(x / 2, y)-f(x, y / 2)+f(x / 2, y / 2)|\right\}^{p} d y d x$

$$
\begin{aligned}
\int_{0}^{a} \int_{0}^{b}\left\{\frac{G(x, y)}{x y}\right\}^{p} d y d x & \\
\leq & \left(\frac{p}{p-1}\right)^{2 p} \int_{0}^{a} \int_{0}^{b}\left\{\frac{|g(x, y)-g(x / 2, y)|}{x y}\right\}^{p} d y d x
\end{aligned}
$$

and

$$
\begin{aligned}
& \int_{0}^{a} \int_{0}^{b}\left\{\frac{H(x, y)}{x y}\right\}^{p} d y d x \\
& \leq\left(\frac{p}{p-1}\right)^{2 p} \int_{0}^{a} \int_{0}^{b}\left\{\frac{|h(x, y)-h(x, y / 2)|}{x y}\right\}^{p} d y d x
\end{aligned}
$$

respectively. The inequalities (9)-(11) can be considered as the two independent variables generalizations of the slight variant of Hardy's inequality given in [4] (Theorem 2).

\section{Proof of Theorem 1}

For $f=0$ the inequality (4) is trivially true. We assume that $f$ is positive and denote by $I$ the integral on the left-hand side of (4). By using Fubini's theorem [1] (p. 18), we observe that

$$
I=\int_{0}^{a}\left(x r_{1}(x)\right)^{-p} I_{1}(x) d x
$$

where

$$
I_{1}(x)=\int_{0}^{b} y^{-p}\left\{\frac{1}{r_{2}(y)} \int_{y / 2}^{y} \frac{r_{2}(t)}{t}\left(\int_{x / 2}^{x} \frac{r_{1}(s) f(s, t)}{s} d s\right) d t\right\}^{p} d y
$$

By keeping $x$ fixed in (13) and integrating by parts, we have the relation 


$$
\begin{aligned}
& \text { (14) } I_{1}(x)=-\frac{b^{-p+1}}{p-1}\left\{\frac{1}{r_{2}(b)} \int_{b / 2}^{b} \frac{r_{2}(t)}{t}\left[\int_{x / 2}^{x} \frac{r_{1}(s) f(s, t)}{s} d s\right] d t\right\}^{p} \\
& +\frac{p}{p-1} \int_{0}^{b} y^{-p+1}\left\{\frac{1}{r_{2}(y)} \int_{y / 2}^{y} \frac{r_{2}(t)}{t}\left[\int_{x / 2}^{x} \frac{r_{1}(s) f(s, t)}{s} d s\right] d t\right\}^{p-1} \\
& \times \frac{1}{r_{2}^{2}(y)}\left[r_{2}(y)\left(\frac{r_{2}(y)}{y} \int_{x / 2}^{x} \frac{r_{1}(s) f(s, y)}{s} d s-\frac{1}{2} \frac{r_{2}(y / 2)}{y / 2} \int_{x / 2}^{x} \frac{r_{1}(s) f(s, y / 2)}{s} d s\right)\right. \\
& \left.-r_{2}^{\prime}(y) \int_{y / 2}^{y} \frac{r_{2}(t)}{t}\left(\int_{x / 2}^{x} \frac{r_{1}(s) f(s, t)}{s} d s\right) d t\right] d y
\end{aligned}
$$

implying

$$
\begin{aligned}
& \int_{0}^{b}\left(1+\frac{p y}{p-1} \frac{r_{2}^{\prime}(y)}{r_{2}(y)}\right) y^{-p}\left\{\frac{1}{r_{2}(y)} \int_{y / 2}^{y} \frac{r_{2}(t)}{t}\left[\int_{x / 2}^{x} \frac{r_{1}(s) f(s, t)}{s} d s\right] d t\right\}^{p} d y \\
\leq & \frac{p}{p-1} \int_{0}^{b}\left\{y^{-p+1}\left[\frac{1}{r_{2}(y)} \int_{y / 2}^{y} \frac{r_{2}(t)}{t}\left(\int_{x / 2}^{x} \frac{r_{1}(s) f(s, t)}{s} d s\right) d t\right]^{p-1}\right. \\
\times & \left.\frac{1}{y r_{2}(y)}\left|r_{2}(y) \int_{x / 2}^{x} \frac{r_{1}(s) f(s, y)}{s} d s-r_{2}(y / 2) \int_{x / 2}^{x} \frac{r_{1}(s) f(s, y / 2)}{s} d s\right|\right\} d y .
\end{aligned}
$$

From (1), (15), using Hölder's inequality with indices $\frac{p}{p-1}, p$, we get

$$
\begin{aligned}
I_{1}(x) \leq & \frac{\alpha p}{p-1} I_{1}^{(p-1) / p}(x)\left[\int _ { 0 } ^ { b } \left\{\frac{1}{y r_{2}(y)} \mid r_{2}(y) \int_{x / 2}^{x} \frac{r_{1}(s) f(s, y)}{s} d s\right.\right. \\
& \left.\left.-r_{2}(y / 2) \int_{x / 2}^{x} \frac{r_{1}(s) f(s, y / 2)}{s} d s \mid\right\}^{p} d y\right]^{1 / p}
\end{aligned}
$$

Dividing both sides of (16) by $I_{1}^{(p-1) / p}(x)$ and raising the result to the $p$-th power, we get

$$
\begin{aligned}
I_{1}(x) \leq & \left(\frac{\alpha p}{p-1}\right)^{p} \int_{0}^{b} y^{-p}\left\{\frac{1}{r_{2}(y)} \mid r_{2}(y) \int_{x / 2}^{x} \frac{r_{1}(s) f(s, y)}{s} d s\right. \\
& \left.-r_{2}(y / 2) \int_{x / 2}^{x} \frac{r_{1}(s) f(s, y / 2)}{s} d s \mid\right\}^{p} d y
\end{aligned}
$$


Substituting (17) in (12) and using Fubini's theorem, we obtain

$$
I \leq\left(\frac{\alpha p}{p-1}\right)^{p} \int_{0}^{b} y^{-p} I_{2}(y) d y
$$

where

$$
\begin{aligned}
\text { (19) } \quad I_{2}(y) & =\int_{0}^{a} x^{-p}\left\{\frac{|m(x, y)|}{r_{1}(x) r_{2}(y)}\right\}^{p} d x \\
\text { (20) } \quad m(x, y) & =r_{2}(y) \int_{x / 2}^{x} \frac{r_{1}(s) f(s, y)}{s} d s-r_{2}(y / 2) \int_{x / 2}^{x} \frac{r_{1}(s) f(s, y / 2)}{s} d s .
\end{aligned}
$$

Now, keeping $y$ fixed in (19), using the relation $|m(x, y)|=m(x, y) \times$ sgn $m(x, y)$ and integrating by parts, we have the equality

$$
\begin{aligned}
& I_{2}(y)=\frac{a^{-p+1}}{-p+1}\left\{\frac{|m(a, y)|}{r_{1}(a) r_{2}(y)}\right\}^{p}+\frac{p}{p-1} \int_{0}^{a} x^{-p+1}\left\{\frac{|m(x, y)|}{r_{1}(x) r_{2}(y)}\right\}^{p-1} \\
& \quad \times \frac{\operatorname{sgn} m(x, y)}{x r_{1}(x) r_{2}(y)}\left[r_{2}(y)\left(r_{1}(x) f(x, y)-r_{1}(x / 2) f(x / 2, y)\right)\right. \\
& \left.-r_{2}(y / 2)\left(r_{1}(x) f(x, y / 2)-r_{1}(x / 2) f(x / 2, y / 2)\right)\right] d x \\
& -\frac{p}{p-1} \int_{0}^{a} x^{-p+1}\left\{\frac{|m(x, y)|}{r_{1}(x) r_{2}(y)}\right\}^{p-1} \frac{r_{1}^{\prime}(x)}{r_{1}(x)} \times \frac{m(x, y) \operatorname{sgn} m(x, y)}{r_{1}(x) r_{2}(y)} d x
\end{aligned}
$$

which implies

$$
\begin{aligned}
& \int_{0}^{a}\left(1+\frac{p x}{p-1} \frac{r_{1}^{\prime}(x)}{r_{1}(x)}\right) x^{-p}\left\{\frac{|m(x, y)|}{r_{1}(x) r_{2}(y)}\right\}^{p} d x \\
& \leq \frac{p}{p-1} \int_{0}^{a}\left[x^{-p+1}\left\{\frac{|m(x, y)|}{r_{1}(x) r_{2}(y)}\right\}^{p-1}\right] \\
& \times\left[\frac{1}{x r_{1}(x) r_{2}(y)} \mid r_{2}(y)\left(r_{1}(x) f(x, y)-r_{1}(x / 2) f(x / 2, y)\right)\right. \\
& \left.-r_{2}(y / 2)\left(r_{1}(x) f(x, y / 2)-r_{1}(x / 2) f(x / 2, y / 2)\right) \mid\right] d x .
\end{aligned}
$$

From (2), (22), using Hölder's inequality with indices $p /(p-1), p$, we get

$$
\begin{aligned}
I_{2}(y) \leq & \frac{\beta p}{p-1} I_{2}^{(p-1) / p}(y)\left[\int _ { 0 } ^ { a } x ^ { - p } \left\{\frac{1}{r_{1}(x) r_{2}(y)}\right.\right. \\
& \times \mid r_{2}(y)\left(r_{1}(x) f(x, y)-r_{1}(x / 2) f(x / 2, y)\right) \\
& \left.\left.-r_{2}(y / 2)\left(r_{1}(x) f(x, y / 2)-r_{1}(x / 2) f(x / 2, y / 2)\right) \mid\right\}^{p} d x\right]^{1 / p} .
\end{aligned}
$$


Dividing both sides of $(23)$ by $I_{2}^{(p-1) / p}(y)$ and raising the result to the $p$-th power, we get

$$
\begin{aligned}
I_{2}(y) \leq & \left(\frac{\beta p}{p-1}\right)^{p} \int_{0}^{a} x^{-p}\left\{\frac{1}{r_{1}(x) r_{2}(y)}\right. \\
& \times \mid r_{2}(y)\left(r_{1}(x) f(x, y)-r_{1}(x / 2) f(x / 2, y)\right) \\
& \left.-r_{2}(y / 2)\left(r_{1}(x) f(x, y / 2)-r_{1}(x / 2) f(x / 2, y / 2)\right) \mid\right\}^{p} d x .
\end{aligned}
$$

Substituting (24) in (18) and using Fubini's theorem, we get (4), which completes the proof of Theorem 1.

We omit the proofs of Theorems 2, 3, being similar to that of Theorem 1 given above.

Re mark 2. We note that the method employed in the proofs of Theorems $1-3$ can be very easily extended to the case of two independent variables in [6] (Theorems 2-5). We do not discuss here the details of these results, as similar to those given in Theorems 1-3.

\section{References}

[1] R. A. Adams, Sobolev Spaces, Academic Press, New York 1995.

[2] G. H. Hardy, Note on a theorem of Hilbert, Math. Z., 6 (1920), 314-317.

[3] G. H. Hardy, J. E. Lit tle wood and G. Poly a, Inequalities, Cambridge University Press 1952.

[4] M. Izumi and S. Izumi, On some inequalities for Fourier series, J. Anal. Math., 21 (1968); 277-291.

[5] N. Levinson, Generalizations of an inequality of Hardy, Duke Math. J., 31 (1964), 389-394.

[6] B. G. Pach patte, On some variants of Hardy's inequality, J. Math. Anal. Appl., 124 (1987), 495-501.

DEPARTMENT OF MATHEMATICS AND STATISTICS

MARATHWADA UNIVERSITY

AURANGABAD 431004 (MAHARASHTRA) INDIA

Received April 21st, 1992. 